\title{
ON THE CANONICAL CUSPS IN COMPLEX HYPERBOLIC SURFACES
}

\author{
JOONHYUNG KIM
}

\begin{abstract}
In this paper, we consider the canonical cusps in complex hyperbolic surfaces. We will classify canonical cusps in complex hyperbolic surfaces and find correspondence between them and 3-dimensional nilpotent groups. This paper is a sequel of our paper [6].
\end{abstract}

\section{Introduction}

Estimating the volumes of cusped complex hyperbolic manifolds has been studied for many years. Many people approached this problem in different ways. See [3], [4], [5], [6], [9]. One intrinsic method to estimate the volumes of cusped complex hyperbolic manifolds is using the fact that canonical cusps are disjoint. In other words, by estimating the volumes of canonical cusps in complex hyperbolic manifolds, one can estimate the volumes of complex hyperbolic manifolds. Especially, in the case of (complex) dimension 2, i.e., in complex hyperbolic surfaces, J. R. Parker got nice results by considering canonical cusps in [9]. In [6], I. Kim and J. Kim, generalized J. R. Parker's method and found better constant in the case of one-ended complex hyperbolic surfaces.

In this paper, we focus on the canonical cusps in cusped complex hyperbolic surfaces. Moreover we also find correspondence between them and 3dimensional nilpotent groups. More concretely, we combine the classification of three dimensional nilmanifolds by K. Dekimpe in [1] with the computation of volumes of canonical cusps for complex hyperbolic manifolds following S. Hersonsky, F. Paulin and J. R. Parker in [3], [6] and [9].

The rest of this paper is organized as follows: In $\S 2$, we briefly discuss some definitions and properties related to the complex hyperbolic space. In $\S 3$, we

Received December 1, 2010; Revised December 22, 2010.

2010 Mathematics Subject Classification. Primary 22E40, 32Q45, 51M10, 57S30.

Key words and phrases. complex hyperbolic manifold, canonical cusp, Heisenberg group, Heisenberg infranilmanifold.

This work was supported by the National Research Foundation of Korea(NRF) grant funded by the Korea government(MEST) (No. 2010-0001194). 
introduce nil geometry briefly, which we use in next chapter. Finally in $\S 4$, we state our main results.

\section{Preliminary}

In this chapter, we introduce some basic materials on complex hyperbolic geometry. We will restrict our attention only in the case of dimension 2 since that is enough in this paper. For more study, we recommend [2].

\subsection{Complex hyperbolic space}

Let $\mathbb{C}^{2,1}$ be a complex vector space of dimension 3 with a Hermitian form of signature $(2,1)$. An element of $\mathbb{C}^{2,1}$ is a column vector $z=\left(z_{1}, z_{2}, z_{3}\right)$. Throughout this paper, we choose the second Hermitian form on $\mathbb{C}^{2,1}$ given by the matrix $J$

$$
J=\left[\begin{array}{lll}
0 & 0 & 1 \\
0 & 1 & 0 \\
1 & 0 & 0
\end{array}\right]
$$

Thus $\langle z, w\rangle=w^{*} J z=z_{1} \overline{w_{3}}+z_{2} \overline{w_{2}}+z_{3} \overline{w_{1}}$, where $w^{*}$ is the Hermitian transpose of $w$.

One model of a complex hyperbolic space $\mathbf{H}_{\mathbb{C}}^{2}$, which matches the second Hermitian form and we will use throughout this paper, is the Siegel domain $\mathfrak{S}$. It is defined by identifying points of $\mathfrak{S}$ with their horospherical coordinates, $z=(\zeta, v, u) \in \mathbb{C} \times \mathbb{R} \times \mathbb{R}_{+}$. For each $u>0$, the horosphere of height $u$ is the subset of $\mathfrak{S}$ given by $H_{u}=\mathbb{C} \times \mathbb{R} \times\{u\}$ and the horoball of height $u$ is $B_{u}=\mathbb{C} \times \mathbb{R} \times(u, \infty)$. The boundary of $\mathfrak{S}$ is given by $H_{0} \cup\left\{q_{\infty}\right\}$, where $q_{\infty}$ is a distinguished point at infinity and $H_{0}=\mathbb{C} \times \mathbb{R} \times\{0\}$.

For each $z=\left(\zeta_{0}, v_{0}\right) \in \mathbb{C} \times \mathbb{R},\left(\zeta_{0}, v_{0}\right) \times \mathbb{R}_{+}$is a geodesic with the other endpoint is $q_{\infty}$. Then there is a canonical projection from $\mathfrak{S}$ to $\mathbb{C} \times \mathbb{R}$ by $(\zeta, v, u) \mapsto(\zeta, v)$ arising from geodesic perspective.

Define a map $\psi: \overline{\mathfrak{S}} \rightarrow \mathbb{P C}^{2,1}$ by

$$
\psi:(\zeta, v, u) \mapsto\left[\begin{array}{c}
\left(-|\zeta|^{2}-u+i v\right) / 2 \\
\zeta \\
1
\end{array}\right] \text { for }(\zeta, v, u) \in \overline{\mathfrak{S}}-\left\{q_{\infty}\right\} ; \psi: q_{\infty} \mapsto\left[\begin{array}{l}
1 \\
0 \\
0
\end{array}\right] .
$$

Then $\psi$ maps $\mathfrak{S}$ homeomorphically to the set of points $z$ in $\mathbb{P C}^{2,1}$ with $\langle z, z\rangle<$ 0 , and maps $\partial \mathfrak{S}$ homeomorphically to the set of points $z$ in $\mathbb{P} \mathbb{C}^{2,1}$ with $\langle z, z\rangle=$ 0 . We write $\psi(\tilde{z})=z$.

The metric on $\mathfrak{S}$ is called the Bergman metric which is given by the distance formula

$$
\cosh ^{2}\left(\frac{\rho(\tilde{z}, \tilde{w})}{2}\right)=\frac{\langle z, w\rangle\langle w, z\rangle}{\langle z, z\rangle\langle w, w\rangle}
$$




\subsection{Heisenberg group}

The boundary of a complex hyperbolic space is identified with the one point compactification of the Heisenberg group. The 3-dimensional Heisenberg group $\mathfrak{N}$ is $\mathbb{C} \times \mathbb{R}$ with the group law

$$
\left(\zeta_{1}, v_{1}\right) \diamond\left(\zeta_{2}, v_{2}\right)=\left(\zeta_{1}+\zeta_{2}, v_{1}+v_{2}+2 \operatorname{Im}\left(\zeta_{1} \overline{\zeta_{2}}\right)\right) .
$$

There is a canonical projection from $\mathfrak{N}$ to $\mathbb{C}$ called the vertical projection given by $\Pi:(\zeta, v) \mapsto \zeta$.

The Heisenberg group carries a natural norm, the Heisenberg norm

$$
|q|=\left(|\zeta|^{4}+v^{2}\right)^{1 / 4}
$$

The Heisenberg group acts on itself by Heisenberg translation. For $(\tau, t) \in \mathfrak{N}$, this translation is

$$
T_{(\tau, t)}:(\zeta, v) \mapsto(\zeta+\tau, v+t+2 \operatorname{Im}(\tau \bar{\zeta}))=(\tau, t) \diamond(\zeta, v) .
$$

A Heisenberg translation by $(0, t)$ for any $t \in \mathbb{R}$ is called the vertical translation by $t$. It is easy to check that the commutator of Heisenberg translations by $(\tau, t)$ and $(\sigma, s)$ is a vertical translation by $4 \operatorname{Im}(\sigma \bar{\tau})$. This fact will be used later.

In the case of dimension 2, the Heisenberg rotation is just $e^{i \theta}$ (In general, the unitary group $U(n-1)$ acts on the Heisenberg group by Heisenberg rotation).

\subsection{Holomorphic isometries}

The holomorphic isometry group of $\mathfrak{S}$ with respect to the Bergman metric is isometric to the projective unitary group $\mathbf{P U}(2,1)$ and it acts on $\mathbb{P C}^{2,1}$ by matrix multiplication.

The action of Heisenberg isometries extends to the Siegel domain, preserving each horosphere and fixing $q_{\infty}$. Some examples of Heisenberg isometries are as follows: The Heisenberg rotation corresponds to the matrix $f \in \mathbf{P U}(2,1)$, and the Heisenberg translation $T_{(\tau, t)}$ to the matrix $g \in \mathbf{P U}(2,1)$, where

$$
f=\left[\begin{array}{ccc}
1 & 0 & 0 \\
0 & e^{i \theta} & 0 \\
0 & 0 & 1
\end{array}\right] \text { and } g=\left[\begin{array}{ccc}
1 & -\tau^{*} & -|\tau|^{2} / 2+i t / 2 \\
0 & I & \tau \\
0 & 0 & 1
\end{array}\right]
$$

When viewed as elements of $\mathbf{P U}(2,1)$, Heisenberg translations are known as pure-parabolic maps and Heisenberg rotations as boundary-elliptic maps. Since every elliptic element in discrete subgroups of $\mathbf{P U}(2,1)$ is of finite order, torsion-free discrete groups cannot contain elliptic elements. A Heisenberg isometry is called screw-parabolic if it is the product of a vertical translation and a Heisenberg rotation. It preserves the fixed-point set of the Heisenberg rotation, called the axis, and acts as a vertical translation there. The screwparabolic map fixing $q_{\infty}$ with axis $(0, v) \subset \mathfrak{N}$, rotation multiplier $e^{i \theta}$, and 
translation length $t$ is

$$
f=\left[\begin{array}{ccc}
1 & 0 & i t / 2 \\
0 & e^{i \theta} & 0 \\
0 & 0 & 1
\end{array}\right]
$$

\subsection{Canonical horoball}

Let $G$ be a discrete subgroup of $\mathbf{P U}(2,1)$ so that the stabiliser $G_{\infty}$ of $q_{\infty}$ contains Heisenberg translations by $(0, t)$ and $\left(\tau, t^{\prime}\right)$, where $t>0$ and $\tau \neq 0$. The horoball $B$ based at $q_{\infty}$ of height

$$
\min \left\{t,\left.|| \tau\right|^{2}+i t / 2 \mid\right\}
$$

is called the canonical horoball at $q_{\infty}$ for $G$.

If $G$ is a discrete, torsion-free subgroup of $\mathbf{P U}(2,1)$ containing a vertical translation by $t>0$ in the stabiliser $G_{\infty}$ of $q_{\infty}$, the canonical horoball at $q_{\infty}$ for $G$ is the horoball $B$ based at $q_{\infty}$ of height $t / 2$. We define the canonical cusp to be $B / G_{\infty}$.

An interesting fact on canonical horoballs is the following:

Proposition 2.1 (Proposition 5.7 in [3] or Proposition 2.4 in [9]). Canonical horoballs at distinct parabolic fixed points are disjoint.

\section{Nil geometry}

This chapter is devoted to introduce nil geometry. The 3-dimensional nil geometry plays an prominent role in this paper, so we briefly introduce some materials which is needed later. For a reference, we recommend [10].

\subsection{Nil 3-manifolds}

As introduced in previous chapter, the 3-dimensional Heisenberg group $\mathfrak{N}$ is $\mathbb{C} \times \mathbb{R}$ with the group law

$$
\left(\zeta_{1}, v_{1}\right) \diamond\left(\zeta_{2}, v_{2}\right)=\left(\zeta_{1}+\zeta_{2}, v_{1}+v_{2}+2 \operatorname{Im}\left(\zeta_{1} \overline{\zeta_{2}}\right)\right)
$$

Then it is easy to see that $\mathfrak{N}$ is a simply connected 2-step nilpotent Lie group, that is $[\mathfrak{N}, \mathfrak{N}]=(0, \mathbb{R})=\mathbb{R}$, which is the center of $\mathfrak{N}$.

There is another way to describe the 3 -dimensional Heisenberg group, which is

$$
\mathfrak{N}=\left\{\left(\begin{array}{lll}
1 & x & t \\
0 & 1 & y \\
0 & 0 & 1
\end{array}\right): x, y, t \in \mathbb{R}\right\}
$$

We can identify them by

$$
\zeta=x+i y, v=2 x y-4 t
$$

or conversely

$$
x=\operatorname{Re}(\zeta), y=\operatorname{Im}(\zeta), t=\frac{\operatorname{Im}\left(\zeta^{2}\right)-v}{4} .
$$


Definition 3.1. A Heisenberg infranilmanifold is a compact manifold $\mathfrak{N} / \pi$, where $\pi$ is a torsion-free discrete cocompact subgroup of $\mathfrak{N} \rtimes C$ for some compact subgroup $C$ of $\operatorname{Aut}(\mathfrak{N})$. Such a group $\pi$ is called an almost Bieberbach group.

Every almost Bieberbach group modelled on $\mathfrak{N}$ is determined by the short exact sequence

$$
1 \rightarrow L \rightarrow \pi \rightarrow \theta \rightarrow 1
$$

where $L=\pi \cap \mathfrak{N}$ and $|\theta|<\infty$. We call $\theta$ the holonomy group of $\pi$. It is well known that $\pi$ contains a cocompact lattice of $\mathfrak{N}$ with index bounded above by a universal constant $I$, i.e., $I$ is the maximal order of the holonomy groups.

Identifying $S^{1}$ with the rotations in the $x y$-plane, since $S^{1}=U(1)$ belongs to the maximal compact subgroup $C$ of $\operatorname{Aut}(\mathfrak{N}), \mathfrak{N} / \Gamma$ is an infranilmanifold, where $\Gamma$ is a discrete subgroup of $\mathfrak{N} \rtimes S^{1}$ which acts freely. When the nil 3manifold is closed, $\mathfrak{N} \cap \Gamma$ must be finite index in $\Gamma$ and $\mathfrak{N} /(\mathfrak{N} \cap \Gamma)$ must be compact.

\subsection{Presentation of nil 3-manifold groups}

In this section, we present a complete list of closed nil 3-manifold groups. Originally, this list was given by K. Dekimpe in [1], but here we follow the notation used by D. B. McReynolds in [8].

(1)

$$
\left\langle a, b, c:[b, a]=c^{p},[c, a]=[c, b]=1\right\rangle \text {, with } p \in \mathbb{N} .
$$

$$
\begin{gathered}
\left\langle a, b, c, \alpha:[b, a]=c^{p},[c, a]=[c, b]=[\alpha, c]=1, \alpha a=a^{-1} \alpha\right. \\
\left.\alpha b=b^{-1} \alpha, \alpha^{2}=c\right\rangle, \text { with } p \in 2 \mathbb{N} .
\end{gathered}
$$

$$
\begin{gathered}
\left\langle a, b, c, \alpha:[b, a]=c^{2 p},[c, a]=[c, b]=[a, \alpha]=1, \alpha c=c^{-1} \alpha,\right. \\
\left.\alpha b=b^{-1} \alpha c^{-p}, \alpha^{2}=a\right\rangle, \text { with } p \in \mathbb{N} .
\end{gathered}
$$

$$
\begin{gathered}
\left\langle a, b, c, \alpha, \beta:[b, a]=c^{2 p},[c, a]=[c, b]=[c, \alpha]=[a, \beta]=1,\right. \\
\beta c=c^{-1} \beta, \alpha a=a^{-1} \alpha c^{p}, \alpha b=b^{-1} \alpha c^{-p} \\
\alpha^{2}=c, \beta^{2}=a, \beta b=b^{-1} \beta c^{-p} \\
\left.\alpha \beta=a^{-1} b^{-1} \beta \alpha c^{-p-1}\right\rangle, \text { with } p \in \mathbb{N} .
\end{gathered}
$$

$$
\begin{gathered}
\left\langle a, b, c, \alpha:[b, a]=c^{p},[c, a]=[c, b]=[c, \alpha]=1, \alpha a=b \alpha\right. \\
\left.\alpha b=a^{-1} \alpha, \alpha^{4}=c\right\rangle, \text { with } p \in 2 \mathbb{N} .
\end{gathered}
$$


$(5-2)$

$$
\begin{gathered}
\left\langle a, b, c, \alpha:[b, a]=c^{2 p},[c, a]=[c, b]=[c, \alpha]=1, \alpha a=b \alpha,\right. \\
\left.\alpha b=a^{-1} \alpha, \alpha^{4}=c^{3}\right\rangle, \text { with } p \in 2 \mathbb{N} .
\end{gathered}
$$

$$
\begin{gathered}
\left\langle a, b, c, \alpha:[b, a]=c^{3 p},[c, a]=[c, b]=[c, \alpha]=1, \alpha a=b \alpha,\right. \\
\left.\alpha b=a^{-1} b^{-1} \alpha, \alpha^{3}=c\right\rangle, \text { with } p \in \mathbb{N} .
\end{gathered}
$$

$$
\begin{gathered}
\left\langle a, b, c, \alpha:[b, a]=c^{3 p},[c, a]=[c, b]=[c, \alpha]=1, \alpha a=b \alpha,\right. \\
\left.\alpha b=a^{-1} b^{-1} \alpha, \alpha^{3}=c^{2}\right\rangle, \text { with } p \in \mathbb{N} .
\end{gathered}
$$

$$
\begin{aligned}
\left\langle a, b, c, \alpha:[b, a]=c^{p},[c, a]=[c, b]=[c, \alpha]\right. & =1, \alpha a=b \alpha c, \\
\left.\alpha b=a^{-1} b^{-1} \alpha, \alpha^{3}=c\right\rangle, & \text { with } p \in \mathbb{N} \text { and } p \equiv 1,2(\bmod 3) .
\end{aligned}
$$

$$
\begin{aligned}
& \left\langle a, b, c, \alpha:[b, a]=c^{p},[c, a]=[c, b]=[c, \alpha]=1, \alpha a=a b \alpha,\right. \\
& \left.\alpha b=a^{-1} \alpha, \alpha^{6}=c\right\rangle \text {, with } p \in \mathbb{N} \text { and } p \equiv 0,4(\bmod 6) .
\end{aligned}
$$

$$
\begin{array}{r}
\left\langle a, b, c, \alpha:[b, a]=c^{p},[c, a]=[c, b]=[c, \alpha]=1, \alpha a=a b \alpha,\right. \\
\left.\alpha b=a^{-1} \alpha, \alpha^{6}=c^{5}\right\rangle, \text { with } p \in \mathbb{N} \text { and } p \equiv 0,2(\bmod 6) .
\end{array}
$$

\section{Main results}

Let $G$ be a discrete, torsion-free, cofinite-volume group of isometries of $\mathbf{H}_{\mathbb{C}}^{2}$, $q_{\infty}$ a parabolic fixed point of $G, G_{\infty}$ its stabiliser in $G, B$ the canonical horoball based at $q_{\infty}$, and $g$ the shortest vertical translation by $t>0$ in $G_{\infty}$. Suppose that $G_{\infty}$ contains a Heisenberg lattice $L$ as a subgroup of index $m$. Then $\Pi(L)$ becomes a lattice in $\mathbb{C}$. Suppose that $\tau$ has the shortest length among all nontrivial elements of $\Pi(L)$ and that $\sigma$ has the shortest length among all elements of $\Pi(L)$ that are not real multiples of $\tau$. Then $\{\tau, \sigma\}$ becomes a basis for $\Pi(L)$ and $\sigma / \tau$ is in the standard fundamental region for the modular group. If we pullback the translations by $\tau$ and $\sigma$ under $\Pi$, they become Heisenberg translations in $L$ by $\left(\tau, t^{\prime}\right)$ and $\left(\sigma, s^{\prime}\right)$ for some $t^{\prime}, s^{\prime} \in \mathbb{R}$. Hence, as mentioned in $\S 2.2$, their commutator is a vertical translation by $4 \operatorname{Im}(\sigma \bar{\tau})=t p$ for some $p \in \mathbb{N}$. Then the volumes of canonical cusps of complex hyperbolic surfaces are expressed as a function of $m$ and $p$. That is,

$$
\operatorname{Vol}_{\mathfrak{S}}\left(B / G_{\infty}\right)=\frac{2 p}{m}
$$


(For the proof, see [6] or [9]). Therefore finding relations between $m$ and $p$ contributes to improve the volume constants.

On the other hand, S. Hersonsky and F. Paulin prove that $I_{2}=6$ (Proposition 5.8 in [3]), so $m \in\{2,3,4,6\}$ because after a vertical projection, the order of the symmetry of the Heisenberg lattice is the same as the plane lattice. That is, the number $m$ is just the order of the rotational symmetry of a lattice in $\mathbb{C}$. It is the so-called crystallographic restriction. Hence $\operatorname{Vol}_{\mathfrak{S}}\left(B / G_{\infty}\right) \geq 1 / 3$ (The equality holds when $m=6$ and $p=1$ ).

In [9], J. R. Parker proves the following theorem, which says that if $m=4$ or 6 , then $p \neq 1$.

Theorem 4.1 (Proposition 5.5 in [9]). Let $G$ be a discrete, torsion-free, cocompact group of Heisenberg isometries. Suppose that $G$ contains a screw-parabolic map $f$ whose rotational part has order $m=4$ or 6 . Let $k$ be the shortest vertical translation in $G$, and let $g$ be a shortest translation in $\Pi(G)$. Now $\left[f g f^{-1}, g\right]$ is a vertical translation, and so $\left[f g f^{-1}, g\right]=k^{ \pm p}$, where $p$ is a positive integer. Then $p \geq 2$.

Then, as corollaries of above theorem, we can estimate the volumes of canonical cusps of complex hyperbolic surfaces and the volumes of cusped complex hyperbolic surfaces. More concretely, we can enlarge the volume constant from $1 / 3$ to $2 / 3$.

Corollary 4.2 (Theorem 5.1 in [9]). Let $G$ be a discrete, torsion-free, cofinitevolume group of isometries of $\mathbf{H}_{\mathbb{C}}^{2}, q_{\infty}$ a parabolic fixed point of $G$, and $G_{\infty}$ its stabiliser in $G$. Let $B$ be the canonical horoball based at $q_{\infty}$. Then

$$
\operatorname{Vol}_{\mathfrak{S}}\left(B / G_{\infty}\right) \geq 2 / 3 .
$$

Corollary 4.3 (Corollary 5.2 in [9]). Let $M$ be a complex hyperbolic 2-manifold with $k$ ends. Then $\operatorname{Vol}_{\mathfrak{S}}(M) \geq 2 k / 3$.

J. R. Parker proves above theorem using the following two lemmas. We will also use them.

Lemma 4.4 (Lemma 5.3 in [9]). Let $G$ be a discrete, torsion-free group of Heisenberg isometries containing the screw-parabolic map $f$ with rotational part of order $m=2,3,4$, or 6 . Let $k$, the vertical translation by $t>0$, be the shortest vertical translation in $G$. Then there is an integer $r$ so that the screw-parabolic map $f k^{-r}$ is also in $G$, has the same rotation multiplier as $f$, and has the property that $\left(f k^{-r}\right)^{m}=k^{ \pm 1}$. In other words, the translation length of $f k^{-r}$ $i s \pm t / m$.

Proof. Since $f^{m}$ is a vertical translation, $f^{m}=k^{l}$ for some $l$. Then the translation length of $f$ becomes $l t / m$. Let $l=r m+d$, where $-m / 2<d \leq m / 2$. Then the translation length of $f k^{-r}$ becomes $l t / m-r t=d t / m$, which is in the interval $(-t / 2, t / 2]$ and the rotational part is the same as that of $f$. Since $G$ is torsion-free, $b$ cannot be zero. Now consider possible values of $b$ case by case. 
1) $m=2 \Rightarrow d=1$.

2) $m=3 \Rightarrow d= \pm 1$.

3) $m=4 \Rightarrow d= \pm 1,2$.

4) $m=6 \Rightarrow d= \pm 1, \pm 2,3$.

It suffices to show that when $m=4, d$ cannot be 2 and when $m=6, d$ cannot be \pm 2 or 3 . In all of these cases, $d$ divides $m$, so we may say $m=d q$. Then the map $\left(f k^{-r}\right)^{q}$ is a screw-parabolic with rotational part of order $d$ and the translation length $t(=d q t / m)$. Thus $\left(f k^{-r}\right)^{q} k^{-1}$ is a Heisenberg rotation, which contradicts to the assumption that $G$ is torsion-free. Hence $d= \pm 1$.

Remark 4.5. Thanks to above lemma, for a given screw-parabolic map $f$ with rotational part of order $m$, we may assume that the translation length of $f$ is $\pm t / m$.

Lemma 4.6 (Lemma 5.4 in [9]). Let $f$ be a screw-parabolic Heisenberg isometry with axis $(o, v) \subset \mathfrak{N}$, rotation multiplier $e^{i \theta}$, and translation length $r \in \mathbb{R}$. Let $g$ be the Heisenberg translation by $(\sigma, s) \in \mathfrak{N}$. Then $g f$ is a screw-parabolic map with axis $\left(\sigma /\left(1-e^{i \theta}\right), v\right)$, rotational multiplier $e^{i \theta}$, and translation length $r^{\prime}=r+s-|\sigma|^{2} \sin \theta /(1-\cos \theta)$.

In [6], I. Kim and J. Kim prove that if $m \in\{2,4,6\}$, then $p$ must be even in the above situation. Especially, it contributes to improve the volume estimate of one-ended complex hyperbolic surfaces (See [6]). Their results are as follows.

Theorem 4.7 (Theorem 4.12 in [6]). Let $G$ be a discrete, torsion-free, cocompact group of Heisenberg isometries. Suppose that $G$ contains a screw-parabolic map $f$ whose rotational part has order $m=2$. Then $p$ must be an even integer, where $p$ is defined as above.

Theorem 4.8 (Theorem 4.13 in [6]). Let $G$ be a discrete, torsion-free, cocompact group of Heisenberg isometries. Suppose that $G$ contains a screw-parabolic map $f$ whose rotational part has order $m=4$ or 6 . Let $k$ be the shortest vertical translation in $G$, and let $g$ be a shortest translation in $\Pi(G)$. Now $\left[f g f^{-1}, g\right]$ is a vertical translation, and so $\left[f g f^{-1}, g\right]=k^{ \pm p}$, where $p$ is a positive integer. Then $p$ must be an even integer.

From now on, we investigate the relations between $m$ and $p$ more concretely and classify all possible cases.

\section{1. $m=2$}

By Lemma 4.4, we may assume that $G$ contains $g$, a Heisenberg translation by $(\sigma, s)$ and $f$, a screw-parabolic with axis $(o, v) \subset \mathfrak{N}$, rotation multiplier -1 , and translation length $r= \pm t / 2$. We know that, in fact, $r=t / 2$, not $-t / 2$ by seeing the proof of Lemma 4.4. We may also assume that $G$ contains $h$, a 
Heisenberg translation by $\left(\tau, s^{\prime}\right)$ so that

$$
[g, h]=\left[\begin{array}{ccc}
1 & 0 & -\sigma^{*} \tau+\tau^{*} \sigma \\
0 & 1 & 0 \\
0 & 0 & 1
\end{array}\right]
$$

which is a vertical translation by $4 \operatorname{Im}(\sigma \bar{\tau})=2 i(\bar{\sigma} \tau-\sigma \bar{\tau})=p t$.

Since $m=2, \Pi\left(G_{\infty}\right)$ is a $(2,2,2,2)$-group and order 2 elliptic conjugacy classes are projections of $f, g f, h f$, and $h g f$ (See Section 5.5, Table 2 in [11] and Section V. D. 9 in [7]). Using Lemma 4.6, we can calculate the translation lengths of them (See the proofs of Theorem 4.12 in [6]). As a summary, the translation length of $f$ is $t / 2$, that of $g f$ is $\left(1 / 2+n_{1}\right) t$, that of $h f$ is $\left(1 / 2+n_{2}\right) t$, and that of $h g f$ is $\left(1 / 2+n_{1}+n_{2}-p / 2\right) t$ for some integers $n_{1}, n_{2}$ and $p \in 2 \mathbb{N}$.

In this case, we may express the group $G$ as

$$
\begin{aligned}
\langle f, g, h, k:[g, h] & =k^{p},[k, f]=[k, g]=[k, h]=1,(g f)^{2}=k^{1+2 n_{1}}, \\
(h f)^{2} & \left.=k^{1+2 n_{2}},(h g f)^{2}=k^{1+2 n_{1}+2 n_{2}-p}, f^{2}=k\right\rangle, \text { with } p \in 2 \mathbb{N},
\end{aligned}
$$

where $k$ is the shortest vertical translation by $t$, and hence $[k, f]=[k, g]=$ $[k, h]=1$. Conjugating $G$ by a Heisenberg translation if necessary, we may assume that $n_{1}=n_{2}=0$. We will show that this group is exactly the same as the nil 3-manifold group (2) in $\S 3.2$. First, replace $(f, g, h, k)$ by $(\alpha, b, a, c)$ respectively. Then $[b, a]=c^{k}, \alpha^{2}=c$ and $[c, a]=[c, b]=[\alpha, c]=1$ are direct. From $(g f)^{2}=(h f)^{2}=f^{2}=k$, we get $(b \alpha)^{2}=(a \alpha)^{2}=\alpha^{2}=c$. Hence, $b \alpha b=a \alpha a=\alpha$, that is $\alpha a=a^{-1} \alpha$ and $\alpha b=b^{-1} \alpha$. In the above group presentation of $G$, we notice that $(h g f)^{2}=k^{1+2 n_{1}+2 n_{2}-p}$ is induced from other relations since $(h g f)^{2}=h g(f h)(g f)=h g\left(h^{-1} k^{1+2 n_{1}} f^{-1}\right)\left(k^{1+2 n_{2}} f^{-1} g^{-1}\right)=$ $h g h^{-1} k^{1+2 n_{1}+2 n_{2}} g^{-1}=h g h^{-1} g^{-1} k^{1+2 n_{1}+2 n_{2}}=k^{-p} k^{1+2 n_{1}+2 n_{2}}=k^{1+2 n_{1}+2 n_{2}-p}$.

Before working in the cases of $m=3$, , or 6 , we set a piece of notation. In those cases, $\Pi\left(G_{\infty}\right)$ is a triangle group, so they have some properties in common. Suppose that $g$ is a Heisenberg translation by $(\sigma, s)$, the axis of $f$ is $(o, v) \subset \mathfrak{N}$, the rotational multiplier of $f$ is $e^{2 i \pi / m}$, and the translation length of $f$ is $r$. Then $f g f^{-1}$ is a Heisenberg translation by $\left(e^{2 i \pi / m} \sigma, s\right)$, so $\left[f g f^{-1}, g\right]$ is a vertical translation by $4|\sigma|^{2} \sin (2 \pi / m)=p t$ (where $t$ is the translation length of $k)$. By Lemma 4.4, we may assume that $r= \pm t / m= \pm 4|\sigma|^{2} \sin (2 \pi / m) / p m$.

\section{2. $m=3$}

Suppose that $m=3$, i.e., $2 \sqrt{3}|\sigma|^{2}=p t$. Then $\Pi\left(G_{\infty}\right)$ is a $(3,3,3)$-triangle group and the generators are $f, g f$, and $f g^{-1}$, where the orders of them are all 3 (See Section 5.5, Table 2 in [11] and Section V. D. 9 in [7]). We already know that $f$ has a rotational multiplier $e^{2 \pi i / 3}$, and a translation length $r= \pm t / 3$. Using Lemma 4.6, we get the rotational multipliers and the translation lengths of screw-parabolic maps $g f$ and $f g^{-1}$. The generator $g f$ has a rotational multiplier $e^{2 \pi i / 3}$, and a translation length $r^{\prime}=r+s-|\sigma|^{2} \sin \left(\frac{2 \pi}{3}\right) /\left(1-\cos \left(\frac{2 \pi}{3}\right)\right)=$ 
$\pm t / 3+s-|\sigma|^{2} / \sqrt{3}= \pm t / 3+s-p t / 6$. On the other hand, $f g^{-1}$ has a rotational multiplier $e^{2 \pi i / 3}$, and a translation length $r^{\prime \prime}= \pm t / 3-s-p t / 6$. Since the orders of $g f$ and $f g^{-1}$ are $3,3 r^{\prime}= \pm t+3 s-p t / 2$ and $3 r^{\prime \prime}= \pm t-3 s-p t / 2$ are integer multiple of $t$. Furthermore, for $G$ to be torsion free, $r^{\prime}, 2 r^{\prime}, r^{\prime \prime}$ and $2 r^{\prime \prime}$ cannot be integer multiples of $t$. If $p=1$, by the facts that $3 r^{\prime}$ and $3 r^{\prime \prime}$ are integer multiples of $t, s=n t / 6(\bmod t)$, where $n$ is an odd integer. If $r=t / 3$, since $r^{\prime}=t / 3+n t / 6-t / 6$ cannot be an integer multiple of $t, n$ must be 3 $(\bmod 6)$. Hence $s=t / 2(\bmod t)$. Similarly, if $r=-t / 3$, then $s=t / 6$ or $5 t / 6$ $(\bmod t)$. When $p=2,3,4,5$, or $6(\bmod 6)$, one can calculate similarly. We write down all cases below.

\begin{tabular}{|l|c|c|}
\hline & $r=t / 3$ & $r=-t / 3$ \\
\hline$p=1$ & $s=t / 2$ & $s=t / 6$ or $5 t / 6$ \\
$p=2$ & $s=t / 3$ or $2 t / 3$ & $s=t$ \\
$p=3$ & $s=t / 2$ & $s=t / 2$ \\
$p=4$ & $s=t$ & $s=t / 3$ or $s=2 t / 3$ \\
$p=5$ & $s=t / 6$ or $5 t / 6$ & $s=t / 2$ \\
$p=6$ & $s=t$ & $s=t$ \\
\hline
\end{tabular}

Like in the case of $m=2$, we may express the group $G$ as

$$
\begin{aligned}
& \left\langle f, g, k:\left[f g f^{-1}, g\right]=k^{p},[k, f]=[k, g]=1, f^{3}=k^{ \pm 1},\right. \\
& \left.(g f)^{3}=k^{ \pm 1+3 s / t-p / 2},\left(f g^{-1}\right)^{3}=k^{ \pm 1-3 s / t-p / 2}\right\rangle, \text { with } p \in \mathbb{N},
\end{aligned}
$$

where $k$ is the shortest vertical translation by $t$, and hence $[k, f]=[k, g]=1$. Then we can identify the group $G$ with the nil 3 -manifold groups (6-1), (6-2) or (6-3) in $\S 3.2$. Precisely, (6-1) is the same as $G$ with $r=t / 3, p=3$ or $6,(6-2)$ is the same as $G$ with $r=-t / 3, p=3$ or 6 , and (6-3) is the same as $G$ with the other cases. For example, if we set $s=-t / 2$, then we can identify $(6-1)$ with $G$ for $r=t / 3$ and $p=3$ by replacing $(a, b, c, \alpha)$ with $\left(g, f g f^{-1}, k, f\right)$. In this case, we may write $G$ as

$$
\begin{gathered}
\left\langle f, g, k:\left[f g f^{-1}, g\right]=k^{3},[k, f]=[k, g]=1, f^{3}=k,\right. \\
\left.(g f)^{3}=k^{-2},\left(f g^{-1}\right)^{3}=k\right\rangle .
\end{gathered}
$$

Then except the relation $\alpha b=a^{-1} b^{-1} \alpha$, the other relations are obtained directly from $G$. From $[k, g]=f^{3} g f^{-3} g^{-1}=1, g f^{2}=f^{3} g f^{-1}$. Hence,

$$
\begin{aligned}
a \alpha b \alpha^{-1} b & =g f\left(f g f^{-1}\right) f^{-1}\left(f g f^{-1}\right) \\
& =\left(g f^{2}\right)\left(g f^{-1}\right)^{2} \\
& =\left(f^{3} g f^{-1}\right)\left(g f^{-1}\right)^{2} \\
& =f^{3}\left(g f^{-1}\right)^{3} \\
& =f^{3} k^{-1}=1 .
\end{aligned}
$$

We notice that $(g f)^{3}=k^{-2}$ is obtained from other relations because

$$
k^{-2}=k^{-3} k=\left[f g f^{-1}, g\right]^{-1} f^{3}
$$




$$
\begin{aligned}
& =\left(g f g f^{-1} g^{-1} f g^{-1} f^{-1}\right) f^{3} \\
& =(g f g)\left(f^{-1} g^{-1} f g^{-1} f^{2}\right) \\
& =(g f g)(f g f) \\
& =(g f)^{3} .
\end{aligned}
$$

Here, we used the equality $f^{-1} g^{-1} f g^{-1} f^{2}=f g f$, which is induced from $k=$ $f^{3}=\left(f g^{-1}\right)^{3}$.

Similarly, if we set $s=-t$, then we can identify (6-1) with $G$ for $r=t / 3$ and $p=6$. For one more example, we consider the case $p=1$ and $r=t / 3$. In this case, if we set $s=-t / 2$ and replace $(a, b, c, \alpha)$ by $\left(g^{-1} f g^{-1} f^{-1}, g, k, f\right)$ respectively, then we can match $G$ to $(6-3)$ with $p \equiv 1(\bmod 3)$.

\section{3. $m=4$}

Suppose that $m=4$, i.e., $4|\sigma|^{2}=p t$. Then $\Pi\left(G_{\infty}\right)$ is a $(2,4,4)$-triangle group and the generators are $f, g f$, and $g f^{2}$, where the orders are 4,4 , and 2, respectively (See Section 5.5, Table 2 in [11] and Section V. D. 9 in [7]). Using Lemma 4.6, we can calculate the translation lengths of them (See the proof of Theorem 4.13 in [6]). Then, the translation length of $f$ is $\pm t / 4$, that of $g f$ is $\pm t / 4+n t-p t / 4$, and that of $g f^{2}$ is $\pm t / 2+n t$ for some integer $n$ and $p \in 2 \mathbb{N}$. In this case, we may express the group $G$ as the following two cases. They correspond to the case $r=t / 4$ and $r=-t / 4(\equiv 3 t / 4 \bmod t)$, respectively.

$$
\begin{aligned}
& \left\langle f, g, k:\left[f g f^{-1}, g\right]=k^{p},[k, f]=[k, g]=1,(g f)^{4}=k^{1+4 l-p},\right. \\
& \left.\left(g f^{2}\right)^{2}=k^{1+2 l}, f^{4}=k\right\rangle, \text { with } p \in 2 \mathbb{N} . \\
& \left\langle f, g, k:\left[f g f^{-1}, g\right]=k^{p},[k, f]=[k, g]=1,(g f)^{4}=k^{3+4 l-p},\right. \\
& \left.\left(g f^{2}\right)^{2}=k^{3+2 l}, f^{4}=k^{3}\right\rangle, \text { with } p \in 2 \mathbb{N} .
\end{aligned}
$$

Conjugating $G$ by a Heisenberg translation if necessary, we may assume that $l=$ 0 . Then we can identify these two groups with the nil 3-manifold groups (5-1) and (5-2) respectively, by replacing $(a, b, c, \alpha)$ in (5-1) or (5-2) by $\left(g, f g f^{-1}, k, f\right)$ in $G$. Let's check it precisely for the first case. First of all, $(g f)^{4}=k^{1-p}$ is induced from other relations since

$$
\begin{aligned}
(g f)^{4} & =g f g(f g)(f g) f=g f g\left(f^{-1} g^{-1} f^{2}\right)\left(f^{-1} g^{-1} f^{2}\right) f \\
& =\left(g f g f^{-1} g^{-1} f g^{-1} f^{-1}\right) f^{4} \\
& =\left[f g f^{-1}, g\right]^{-1} f^{4} \\
& =k^{-p} k=k^{1-p} .
\end{aligned}
$$

We used $f g=f^{-1} g^{-1} f^{2}$ from $\left(g f^{2}\right)^{2}=k=f^{4}$. Furthermore $\left[f g f^{-1}, g\right]=k^{p}$, $[k, f]=[k, g]=1$ and $f^{4}=k$ are obtained directly from (5-1), and $\alpha a=b \alpha$ is just $f g=f g$. Finally, $\alpha b=a^{-1} \alpha$ is changed by $f^{2} g f^{-1}=g^{-1} f$, which is $\left(g f^{2}\right)^{2}=f^{4}=k$. For the second case, it is almost the same, but we have to notice that $[b, a]=c^{2 p}$, not $[b, a]=c^{p}$. Hence to match exactly in second case, 
$p$ must be changed by the integer multiple of 4 , not the integer multiple of 2 . Therefore we can conclude that if the translation length of $f$ is $-t / 4, p$ must be an integer multiple of 4 .

\section{4. $m=6$}

Finally suppose that $m=6$, i.e., $2|\sigma|^{2} \sqrt{3}=p t$. Then $\Pi\left(G_{\infty}\right)$ is a $(2,3,6)$ triangle group and the generators are $f, g f^{2}$, and $g f^{3}$, where the orders are 6,3 , and 2, respectively (See also Section 5.5, Table 2 in [11] and Section V. D. 9 in [7] as above). As we see the proof of Theorem 4.13 in [6], the translation length of $f$ is $\pm t / 6$, that of $g f^{2}$ is $\pm t / 3+n t-p t / 6$, and that of $g f^{3}$ is $\pm t / 2+n t$ for some integer $n$ and $p \in 2 \mathbb{N}$. Furthermore, for $G$ to be torsion free, $\pm t / 3+n t-p t / 6$ and $2( \pm t / 3+n t-p t / 6)= \pm 2 t / 3+2 n t-p t / 3$ cannot be integer multiple of $t$. Hence, if $r=t / 6$, then $p=0$ or $4(\bmod 6)$, and if $r=-t / 6$, then $p=0$ or 2 $(\bmod 6)$.

Like above cases, we may express the group $G$ as following two cases.

They correspond to the case $r=t / 6$ and $r=-t / 6(\equiv 5 t / 6 \bmod t)$, respectively.

$$
\begin{gathered}
\left\langle f, g, k:\left[f g f^{-1}, g\right]=k^{p},[k, f]=[k, g]=1,\left(g f^{2}\right)^{3}=k^{1+3 l-p / 2},\right. \\
\left.\left(g f^{3}\right)^{2}=k^{1+2 l}, f^{6}=k\right\rangle, \text { with } p \equiv 0,4(\bmod 6), \\
\left\langle f, g, k:\left[f g f^{-1}, g\right]=k^{p},[k, f]=[k, g]=1,\left(g f^{2}\right)^{3}=k^{5+3 l-p / 2},\right. \\
\left.\left(g f^{3}\right)^{2}=k^{5+2 l}, f^{6}=k^{5}\right\rangle, \text { with } p \equiv 0,2(\bmod 6) .
\end{gathered}
$$

Conjugating $G$ by a Heisenberg translation if necessary, we may assume that $l=0$. Then we can identify these two groups with the nil 3 -manifold groups (7-1) and (7-2) respectively, by replacing $(a, b, c, \alpha)$ by $\left(f g^{-1} f^{-1}, g, k, f\right)$. For the first case, first of all, $[b, a]=c^{p}$ is changed by $\left[g, f g^{-1} f^{-1}\right]=k^{p}$. Here $\left[g, f g^{-1} f^{-1}\right]=k^{p}$ is equivalent to $\left[f g f^{-1}, g\right]=k^{p}$ because

$$
\begin{aligned}
& {\left[g, f g^{-1} f^{-1}\right]=g f g^{-1} f^{-1} g^{-1} f g f^{-1}=k^{p} } \\
\Leftrightarrow & \left(g f g^{-1} f^{-1} g^{-1}\right)\left(f g f^{-1}\right) k^{-p}=1 \\
\Leftrightarrow & \left(g f g^{-1} f^{-1} g^{-1}\right) k^{-p}\left(f g f^{-1}\right)=1 \\
\Leftrightarrow & \left(g f g^{-1} f^{-1} g^{-1}\right)^{-1}=k^{-p}\left(f g f^{-1}\right) \\
\Leftrightarrow & \left(f g f^{-1}\right)\left(g f g^{-1} f^{-1} g^{-1}\right)=k^{p} \\
\Leftrightarrow & {\left[f g f^{-1}, g\right]=k^{p} . }
\end{aligned}
$$

Next, $[k, f]=[k, g]=1$ and $f^{6}=k$ are direct from $[c, a]=[c, b]=[c, \alpha]=1$ and $\alpha^{6}=c$. Furthermore $\alpha b=a^{-1} \alpha$ is just changed by $f g=f g$. Finally we have only to consider $\alpha a=a b \alpha$, which is changed by $f\left(f g^{-1} f^{-1}\right)=\left(f g^{-1} f^{-1}\right) g f$, that is $g^{-1} f g f g^{-1}=f^{2}$. Before considering it, we consider relations in $G$. By relations in $G$, we get the following equality.

$$
k^{p}=\left(g f^{2}\right)^{-6}\left(g f^{3}\right)^{6} f^{-6}
$$




$$
\begin{aligned}
& =\left(f^{-2} g^{-1}\right)^{5}\left(f^{-2} g^{-1} g f^{3}\right) g f^{3}\left(g f^{3} g\right) f^{3}\left(g f^{3} g\right) f^{3} f^{-6} \\
& =\left(f^{-2} g^{-1}\right)^{5}(f) g f^{3}\left(f^{3}\right) f^{3}\left(f^{3}\right) f^{3} f^{-6} \\
& =\left(f^{-2} g^{-1}\right)^{5} f g f^{9} \\
& =f^{4}\left(g^{-1} f^{-2}\right)^{4} g^{-1} f\left(g f^{3}\right) \\
& =f^{4}\left(f^{-3} g f\right)^{4} g^{-1} f\left(f^{3} g^{-1}\right) \\
& =f g\left(f^{-2} g\right)^{3} f g^{-1} f^{4} g^{-1} \\
& =f^{7} g\left(f^{-2} g\right)^{3} f g^{-1} f^{-2} g^{-1} \\
& =\left[f g f^{-1}, g\right]=f g f^{-1} g f g^{-1} f^{-1} g^{-1}
\end{aligned}
$$

Hence,

$$
\begin{aligned}
& f^{6} g f^{-2} g f^{-2} g f^{-2} g f g^{-1} f^{-1}=g f^{-1} g f g^{-1} \\
\Leftrightarrow & f^{-1} g f^{-2} g f^{-2} g f g^{-1} f^{5}=g f g^{-1} \\
\Leftrightarrow & g\left(f^{-2} g\right) f^{-2} g f g^{-1} f^{5}=f g f g^{-1} \\
\Leftrightarrow & g\left(f g^{-1} f^{-3}\right) f^{-2} g f g^{-1} f^{5}=f g f g^{-1} \\
\Leftrightarrow & g f g^{-1} f^{-5} g f g^{-1} f^{5}=f g f g^{-1} \\
\Leftrightarrow & g\left(f g^{-1} f g f g^{-1}\right) f^{-1} g f^{-1} g^{-1} f^{-1}=1 \\
\Leftrightarrow & f\left(g^{-1} f g f g^{-1}\right)=\left(g^{-1} f g f g^{-1}\right) f .
\end{aligned}
$$

Therefore, from $g^{-1} f g f g^{-1}=f^{2}$, this equality holds.

Remark 4.9. In the list of closed nil 3-manifold groups, (1) corresponds to the case $m=1$. That is, there is no screw-parabolic element in $G$, or $G$ is composed of only Heisenberg translations. Moreover, (3) and (4) correspond to the cases whose holonomy groups are not in $U(n)$, but in $\mathbb{Z}_{2} \times \mathbb{Z}_{2}$, and we need not consider them here (See [5]).

Remark 4.10. In [5], Y. Kamishima proves that $m$ must be 1 on one-ended complex hyperbolic surfaces. That is, there is no lattice subgroup.

Acknowledgement. The author thanks Hyuk Kim, Inkang Kim, and J. R. Parker for encouragements and useful discussions, as well as an anonymous referee for pointing out some inaccuracies in the first draft of the manuscript.

\section{References}

[1] K. Dekimpe, Almost-Bieberbach Groups: Affine and Polynomial Structures, Lecture Notes in Mathematics, 1639. Springer-Verlag, Berlin, 1996.

[2] W. M. Goldman, Complex Hyperbolic Geometry, Oxford Univ. Press, 1999.

[3] S. Hersonsky and F. Paulin, On the volumes of complex hyperbolic manifolds, Duke Math. J. 84 (1996), no. 3, 719-737.

[4] J.-M. Hwang, On the volumes of complex hyperbolic manifolds with cusps, Internat. J. Math. 15 (2004), no. 6, 567-572. 
[5] Y. Kamishima, Nonexistence of cusp cross-section of one-cusped complete complex hyperbolic manifolds II, Int. Math. Forum 2 (2007), no. 25-28, 1251-1258.

[6] I. Kim and J. Kim, On the volumes of canonical cusps of complex hyperbolic manifolds, J. Korean Math. Soc. 46 (2009), no. 3, 513-521.

[7] B. Maskit, Kleinian Groups, Grundlehren der Mathematischen Wissenschaften, 287. Springer-Verlag, Berlin, 1988.

[8] D. B. McReynolds, Peripheral separebility and cusps of arithemetic hyperbolic orbifolds, Algebr. Geom. Topol. 4 (2004), 721-755.

[9] J. R. Parker, On the volumes of cusped, complex hyperbolic manifolds and orbifolds, Duke Math. J. 94 (1998), no. 3, 433-464.

[10] P. Scott, The geometries of 3-manifolds, Bull. London Math. Soc. 15 (1983), no. 5, 401-487.

[11] W. Thurston, The geometry and topology of 3-manifolds, preprint, 1991.

Department of Mathematics

KONKUK UNIVERSITY

SEOUl 143-701, Korea

E-mail address: calvary@snu.ac.kr 